\title{
Lobular Breast Cancer Metastasis to the Colon, the Appendix and the Gallbladder
}

\author{
Rocio Molina-Barea ${ }^{a}$ Rosa M. Rios-Peregrina ${ }^{b}$ Mahmoud Slim $^{c} \quad$ Elena P. Calandre \\ Maria D. Hernández-García ${ }^{a}$ José A. Jimenez-Riosa
}

a Servicio de Cirugía General y del Aparato Digestivo, Hospital Universitario 'San Cecilio', Granada, Spain

b Servicio de Anatomía Patológica, Hospitales Universitarios de Granada, Spain

${ }^{\mathrm{c}}$ Instituto de Neurociencias, Universidad de Granada, Spain

\section{Established Facts}

- The most common sites of metastasis in breast cancer are the lungs, bones, brain, and liver.

- Gastrointestinal metastases are rarely encountered.

\section{Novel Insights}

- This case describes metastases of breast cancer to the gallbladder, which is an exceptional observation, and cecal appendix infiltration, which has not been reported previously.

- Patients with past history of breast cancer displaying biliary colic symptoms should be thoroughly investigated to rule out potential gallbladder metastases.

\section{Keywords}

Breast cancer · Metastasis · Colon · Appendix · Gallbladder

\section{Summary}

Background: Metastases of lobular breast cancer are commonly encountered at the level of lungs, bones, brain and liver, whereas lesions in the gastrointestinal tract are rarely seen. Case Report: A case of a patient with metastases in the right colon and gallbladder originating from an invasive lobular carcinoma is described. Conclusion: Adequate diagnostic procedures should be performed in patients with a history of breast cancer and who show gastrointestinal symptoms to rule out the potential presence of gastrointestinal metastases.

\section{Introduction}

Lobular breast cancer, the second most frequent type of invasive breast cancer, has a prevalence rate of $5-15 \%$. The most common sites of metastases for such carcinomas are the lungs, bones, brain and liver, whereas lesions in the gastrointestinal tract are rarely encountered [1]. Evidence in the literature concerning gastrointestinal metastases is limited, and mainly based on descriptive studies of autopsy series, clinical case reports and small series of patients [2]. Within the gastrointestinal tract, the stomach and the small intestine are the most common metastatic sites of lobular carcinoma, whereas large intestine metastases are very rare. The presence of a solitary metastasis at this level can easily be confused with a primary colonic neoplasm [3]. Moreover, it is important to

\section{KARGER \\ Fax +49 7614520714 \\ Information@Karger.com}

www.karger.com

\section{(c) 2014 S. Karger GmbH, Freiburg}

1661-3791/14/0096-0428\$39.50/0

Accessible online at:

www.karger.com/brc
Prof. Elena P. Calandre M.D.

Instituto de Neurociencias

Universidad de Granada

Avenida de Madrid 11, 18012 Granada, Spain

epita@ugr.es 
take into consideration that the presenting symptoms and signs might be very nonspecific, contributing to a delayed diagnosis and subsequent worsened prognosis.

We report the case of a patient with metastases in the right colon and gallbladder originating from an invasive lobular carcinoma that was discovered when she underwent urgent surgery under the presumptive diagnosis of complicated biliary colic.

\section{Case Report}

The patient was a 62-year-old female with a past medical history of left mastectomy and axillary dissection subsequent to lobular carcinoma in 2006. The original tumor was classified as an infiltrating lobular carcinoma that was estrogen receptor (ER) positive $(+/++)$, and progesterone receptor negative. Immunohistochemical markers indicated that the carcinoma was c-erbB-2 (HER2/neu) negative, and p53 positive (1\%). As the ER positivity was low, she did not receive anti-estrogen therapy, and was treated with a combination of adriamycin, cyclophosphamide and docetaxel plus axillary radiotherapy. She underwent regular outpatient revisions for tumor metastasis until June 2010, always with negative findings. In January 2011, the patient showed symptoms clinically compatible with biliary colic attacks that obliged her to seek medical treatment at an emergency department several times within 3 weeks. Following these repeated episodes, and after the ultrasound detection of abundant calculi in the gallbladder in addition to analytical findings that only demonstrated discrete leukocytosis, she received surgery under a presumptive diagnosis of complicated biliary colic.

During the surgical procedure, a large tumor was found in the hepatic flexure of the colon. The tumor had a major axis diameter of $10 \mathrm{~cm}$, and it was adhered to the retroperitenium, the duodenum and the cava. The presumptive diagnosis after laparotomy was primary colonic neoplasm associated to chronic lithiasic cholecystopathy. However, the results of the anatomopathological analysis of the colon were consistent with metastatic lobular breast cancer infiltrating the serosal layer, muscular and mucosa; both the gallbladder and the cecal appendix were also infiltrated (figs. 1 and 2). Immunohistochemical analyses were positive for cytokeratine 7 and ER, and negative for the remaining receptors tested including the CDX2 type (fig. 3). Figures 1 and 2 show the infiltrating metastatic cells in the appendix and the gallbladder, respectively. After surgery, she was treated with arimidex and paclitaxel. However, despite the treatment, the patient died 12 months later, as a result of an intestinal obstruction secondary to peritoneal carcinomatosis caused by the progression of the metastatic process.

\section{Discussion}

In the present case, the patient had a solitary tumor that was initially suspected to be a primary colonic neoplasm. However, following anatomopathological analysis of the tumor, it was recognized as a metastasis of her previous breast cancer. Histological diagnosis of metastatic lesions can be challenging and immunohistochemistry is required for a precise diagnosis. In the present case, the tumor cells were positive for ER, corresponding to the results for her primary tumor. Immunostaining for CDX2 facilitated the differential diagnosis between primary or metastatic carcinoma in the bowel, as this biomarker is consistently positive in the primary lesions and negative in most of the metastatic lesions. Exceptions are endometrioid carcinoma of the ovary and cervical adenocarcinoma, among which some neoplasms can be positive for this marker [4].

A particularly interesting aspect of this case was the involvement of not only the colon, but also the cecal appendix
Fig. 1. Metastatic cell infiltration of the cecal appendix. The appendix wall appears to be infiltrated by homogeneous and relatively small neoplastic cells in a pagetoid infiltration pattern of single cells.

Fig. 2. Neoplastic infiltration of the gallbladder wall, without mucosal involvement. The tumor cells are homogenous and relatively small with a single-file infiltrating pattern of cells.
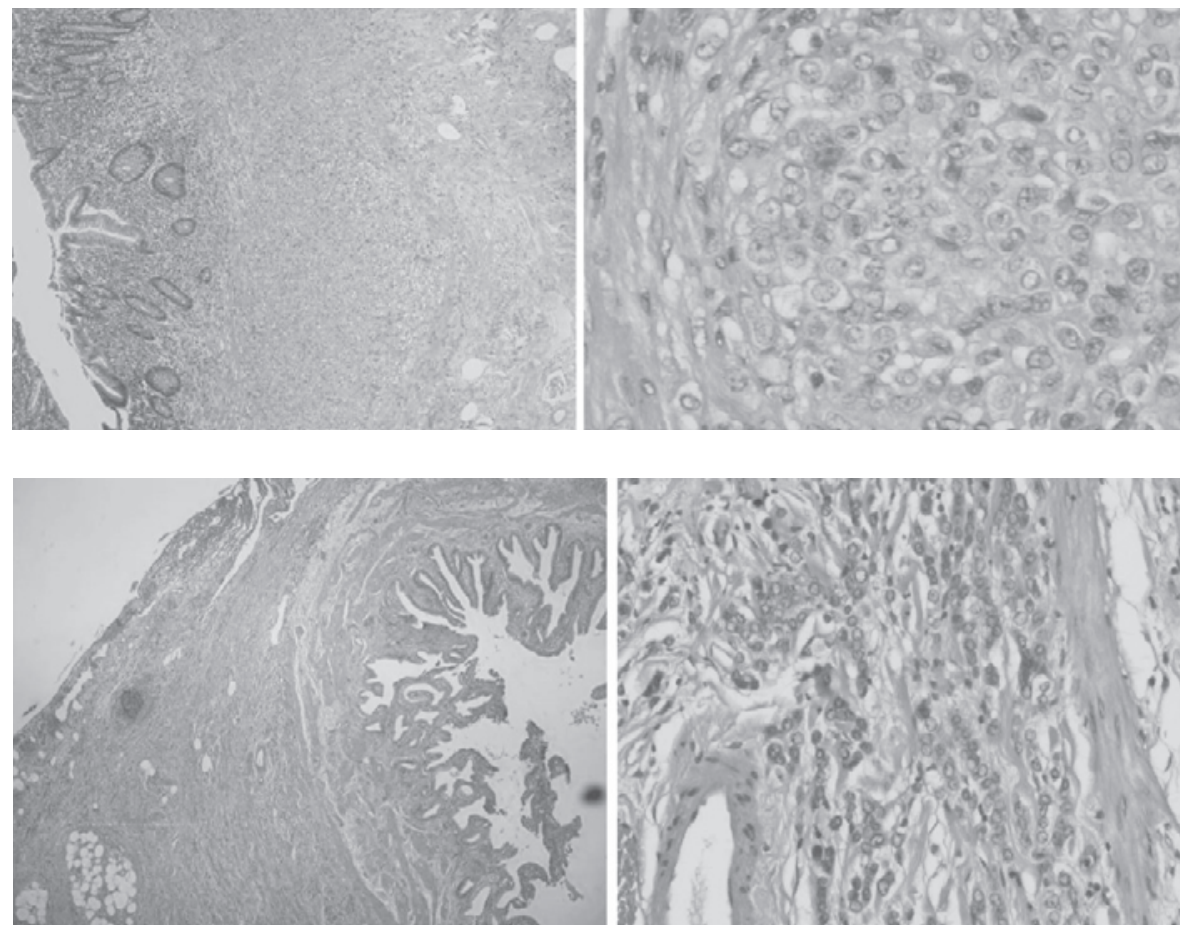
Fig. 3. Tumor cells are positive for anti-estrogen receptors, as in the case of the primary tumor.
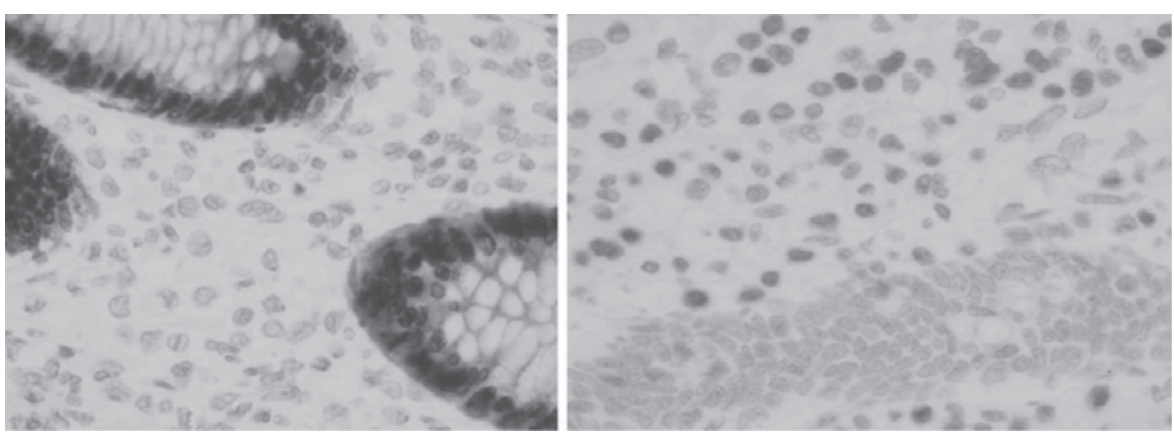

and the gallbladder. Regarding the involvement of the appendix, to our knowledge, no metastasis of a lobular breast carcinoma has been reported at this level.

Only very few cases of metastatic infiltration to the gallbladder have been described previously [5]. In a series of 167 autopsies of patients with breast cancer, metastasis to the gallbladder was seen only in $6.6 \%$ of cases [6]. The clinical presentation of such metastases can be suggestive of acute cholecystitis, especially in the presence of abdominal pain and leukocytosis, as noted in the present case [5, 7]. For our patient, these symptoms appeared 5 years following the surgical treatment of the primary tumor, and she had been asymptomatic at the follow-up revisions, lacking any clinical history suggestive of either constitutional symptoms or potential gastrointestinal tumor pathology. In the majority of case reports dealing with colorectal metastasis of breast cancer, the average interval between the manifestation of the primary tumor and the appearance of metastatic symptoms was $6-8$ years [8].

These patients are characterized by a poor prognostic profile, and few survive longer than 2 years following their diagnosis with gastrointestinal metastasis [2]. Surgical treatment of the gastrointestinal metastasis of breast cancer does not seem to increase the survival rates, except in a subgroup of patients who exclusively suffer from this form of metastasis [9]. However, cases with an up to 9-year survival period have been described in the literature; this fact emphasizes the importance of a timely diagnosis and a prompt initiation of therapy [8]. It is therefore crucial to avoid potential misdiagnosis; in the case series conducted by McLemore et al. [10], which included 73 patients, $21 \%$ of the cases were initially misdiagnosed and $11 \%$ had a correct diagnosis only after the use of exploratory laparotomy.

In conclusion, we believe that patients with a history of breast cancer and who show gastrointestinal symptoms, even if these are nonspecific, should undergo appropriate diagnostic procedures, such as colonoscopy, computed tomographyscan and/or magnetic resonance imaging, to rule out the potential presence of gastrointestinal metastases.

\section{Disclosure Statement}

The authors declare no conflicts of interest.

\section{References}

1 Arrangoiz R, Papavasiliou P, Dushkin H, Farma JM: Case report and literature review: Metastatic lobular carcinoma of the breast an unusual presentation. Int J Surg Case Rep 2011;2:301-305.

2 Bamias A, Baltayiannis G, Kamina S: Rectal metastases from lobular carcinoma of the breast: Report of a case and literature review. Ann Oncol 2001;12:715-718.

3 Dhar S, Kulaylat MN, Gordon K, et al.: Solitary papillary breast carcinoma metastasis to the large bowel presenting as primary colon carcinoma: Case report and review of the literature. Am Surg 2003;69:799-803.
4 Birla R, Mahawar KK, Orizu M, et al.: Caecal metastasis of breast cancer presenting as intestinal obstruction. World J Surg Oncol 2008;6:47.

5 Doval DC, Bhatia K, Pavithran K, et al.: Breast carcinoma with metatasis to the gallbladder: An unusual case report with short review of literature. Hepatobiliary Pancreat Dis Int 2006;5:305-307.

6 Abrahams HL, Spiro R, Goldstein N: Metastases in carcnioma, analysis of 1000 autopsied cases. Cancer 1950;3:74-85.

7 Essola B, Malvaux P, Landenne J, et al.: Métastase vésiculaire d'un carcinome mamaire: Une nouvelle observation. Rev Med Brux 2012;33:171-175.
8 López Deogracias M, Flores Jaime L, AriasCamisón I, et al.: Rectal metastasis from lobular breast carcinoma 15 years after primary diagnosis. Clin Transl Oncol 2010;12:150-153.

-9 Bustamante-Balen M, Navarro-Hervas M, Cuevas JM, Garcia-Diez JV: [Colon metastasis of a lobular breast carcinoma]. Rev Esp Enferm Dig 2008;100: 249-250.

10 McLemore EC, Pockaj BA, Reynolds C, et al.: Breast cancer: Presentation and intervention in women with gastrointestinal metastases and carcinomatosis. Ann Surg Oncol 2005;12:886-894. 